\title{
CONVOLUTIONS WITH RATIONAL KERNELS ${ }^{1}$
}

\author{
JEROME BLACKMAN
}

1. The problem. In recent years a great deal of attention has been paid to the convolution transform.

$$
f(u)=\int_{-\infty}^{\infty} k(u-x) d \alpha(x) .
$$

Recently Pollard [2] considered the case $k(x)=\left(1+x^{2}\right)^{-1}$ and solved (1) for $\alpha(x)$ under very weak conditions assuring convergence of the integral in (1). Our purpose is to treat the case where $k(x)$ is a rational function. More specifically we shall assume the following five conditions.

(i) $k(x)=p(x) / q(x)$ where $p(x)$ and $q(x)$ are polynomials of degree $p$ and $q$ respectively. The distance $d$ from the set of zeros of $q(x)$ to the real axis is positive.

(ii) $q-p=n \geqq 1$.

(iii) $K(x)=(2 \pi)^{-1 / 2} \int_{-\infty}^{\infty} k(t) e^{i x t} d t \neq 0$ for any real value of $x$.

(iv) $\alpha(x)$ is locally of bounded variation and $\alpha(0)=2^{-1}(\alpha(0+)$ $+\alpha(0-))=0$.

(v) For some $u_{0}$ with $\left|\operatorname{Im}\left(u_{0}\right)\right|<d$,

$$
\lim _{A, B \rightarrow \infty} \int_{-A}^{B} k\left(u_{0}-x\right) d \alpha(x)
$$

exists.

Under these conditions we shall present an inversion formula for (1). Although our method allows us to treat a wider class of kernels with shorter proofs, the results are not as elegant as those obtained by Pollard using operational methods. At present the relation between his solution and ours in the special case $k(x)=\left(1+x^{2}\right)^{-1}$ is not clear. Our work is not covered by the work of Hirschman and Widder [1].

It should be noted that once the restriction to rational kernels is made conditions (i) - (v) are very natural for this problem. Indeed (i), (iv), and (v), are needed to insure the existence of (1) in a rather weak sense while (ii) and (iii) are needed to insure the existence of $K(x)$

Presented to the Society, April 20, 1956; received by the editors November 10 , 1955 and, in revised form, March 9, 1956 and April 11, 1956.

1 The research on this paper was supported by the U.S. Air Force under Contract No. AF 18 (600)-685 monitored by The Office of Scientific Research. 
and its nonvanishing. The latter condition is, as is well known, necessary for the uniqueness of the solution of (1).

The results will be contained in three theorems. Let

$$
\begin{aligned}
\beta_{t}^{m}(s)= & (2 \pi)^{-3 / 2} \int_{-\infty}^{\infty} f^{(4)}(u) d u \int_{-\infty}^{\infty}(-i y)^{m-4} \\
& \cdot \exp \left(-t y^{2}-i y(s-u)\right) K(y)^{-1} d y,
\end{aligned}
$$

with $t>0$ and $m=2^{n+4}+n+2$, and let

$$
\tilde{\alpha}_{t}(s)=(4 \pi t)^{-1 / 2} \int_{-\infty}^{\infty} \exp \left(-(s-y)^{2} / 4 t\right) d \alpha(y) .
$$

Our first result is

TheOREM 1. $\beta_{t}^{m}(s)=\tilde{\alpha}_{t}^{(m)}(s)$.

Once this is known it is necessary to determine $\tilde{\alpha}_{t}(s)$ from its $m$ th derivative. This is complicated by the fact that the parameter $t$ appears and we want to know $\tilde{\alpha}_{t}(s)$ for all $t>0$. That this is possible is shown in the next theorem.

TheOREM 2. For each $t>0$ choose a fixed mth integral of $\beta_{t}^{m}(s)$, say $\beta_{t}(s)$. Then there is a unique function $p_{t}^{m-1}(s)$ where $p_{t}^{m-1}(s)$ is a polynomial of degree $m-1$ for each $t$, such that

$$
\beta_{t}(s)=\tilde{\alpha}_{t}(s)+p_{t}^{m-1}(s) .
$$

In the proof of Theorem 2 a specific method for constructing $p_{t}^{m-1}(s)$ will be given. Once $\tilde{\alpha}_{t}(s)$ has been found the solution is contained in our final result.

THEOREM 3. The unique solution of (1) under conditions (i)-(v) is given by

$$
\lim _{t \rightarrow 0+} \int_{0}^{s} \tilde{\alpha}_{t}(s) d s=2^{-1}(\alpha(s+)+\alpha(s-)) .
$$

2. Proofs of the theorems. We start with two lemmas whose proofs are easily obtained by slightly modifying the proofs of the analogous lemmas in [2].

LEMMA 1.

$$
\lim _{A, B \rightarrow \infty} \int_{-A}^{B} k(u-x) d \alpha(x)
$$

converges uniformly in every compact subset of $|\operatorname{Im}(u)|<d$. 
Lemma 2.

$$
\alpha(t)=o\left(t^{n}\right) .
$$

It follows from Lemma 1 that

(4) $f^{(j)}(u)=\lim _{A, B \rightarrow \infty} \int_{-A}^{B} k^{(j)}(u-x) d \alpha(x)$ for $|\operatorname{Im}(u)|<d$ and $j \geqq 0$.

Also a simple computation shows that

$$
k^{(j)}(x)=O\left(|x|^{-n-j}\right) \text { as }|x| \rightarrow \infty, \quad \text { for } j \geqq 0,
$$

and that therefore $x^{n+2} k^{(4)}(x) \in L_{1}$. Since $(-i y)^{4} K(y)$ is the Fourier transform of $k^{(4)}(x)$ we can conclude that $y^{4} K(y)$ has $n+2$ continuous derivatives.

A simple estimate for the size of $K(x)$ can be obtained by observing that the integral of (iii) can always be evaluated by contour integration from which it follows that

$$
K(x)= \begin{cases}\sum \sum c_{i j} x^{j} \exp \left(z_{i} x\right) & \text { for } x \geqq 0, \\ \sum \sum c_{i j}^{\prime} x^{j} \exp \left(z_{i}^{\prime} x\right) & \text { for } x<0,\end{cases}
$$

where the summations are over a finite number of terms only. Using this and (iii) it follows that for some $c_{1}$ and $c_{2}$.

$$
|K(x)| \geqq c_{1} \exp \left(c_{2}|x|\right)
$$

so that

$$
y^{m} \exp \left(-t y^{2}\right) K(y)^{-1} \in L_{1}
$$

for every $t>0$ and all $m \geqq 0$.

For what follows we need the inequality

$$
\left|\int_{-A}^{A} k^{(j)}(u-x) d \alpha(x)\right| \leqq c\left(1+\left|u^{n}\right|\right)
$$

for some $c$ independent of $A$ and all $j \geqq 0$. If we integrate by parts and use Lemma 2 and (5) we find

$$
\begin{aligned}
& \left|\int_{-A}^{A} k^{(j)}(u-x) d \alpha(x)\right| \leqq c_{3} A^{n}\left(1+|u-A|^{n+j}\right)^{-1} \\
& \quad+c_{3} A^{n}\left(1+|u+A|^{n+j}\right)^{-1}+c_{4} \int_{-\infty}^{\infty} x^{n}\left(1+|u-x|^{n+j+1}\right)^{-1} d x
\end{aligned}
$$

Letting $u-A=t$, we have 


$$
\begin{aligned}
\left|c_{3} A^{n}\left(1+|u-A|^{n+j}\right)^{-1}\right| & \leqq \sup _{i} c_{3}|u-t|^{n}(1+|t|)^{-n-1} \\
& \leqq c_{3}^{\prime} u^{n}+c_{3}^{\prime} .
\end{aligned}
$$

The second term in (7) is treated the same way and a change of variable in the integral on the right of (7) shows that essentially the same inequality holds thereby proving (6).

If we now combine (2) and (4) we see that

$$
\begin{aligned}
\beta_{t}^{m}(s)= & (2 \pi)^{-3 / 2} \int_{-\infty}^{\infty} d u \lim _{A \rightarrow \infty} \int_{-A}^{A} k^{(4)}(u-x) d \alpha(x) \\
& \cdot \int_{-\infty}^{\infty}(-i y)^{m-4} K(y)^{-1} \exp \left(-t y^{2}-i y(s-u)\right) d y .
\end{aligned}
$$

A direct estimate shows that since $y^{4} K(y)$ has $n+2$ continuous derivatives,

$$
(-i y)^{m-4} K(y)^{-1} \exp \left(-t y^{2}\right)
$$

has $n+2$ derivatives all in $L_{1}$. Therefore the innermost integral in (8) is $O\left(|s-u|^{-n-2}\right)$. Using (6) we see that the outer integral in (8) is uniformly convergent in $A$ so that we may interchange the limit on $A$ and the outer integral.

Since the inner integral is $O\left(|s-u|^{-n-2}\right)$ and $k^{(4)}(u-x)$ is a rational function which is $O(|u-x|-n-4)$ we conclude that

$$
\int_{-\infty}^{\infty} d u\left|k^{(j)}(u-x)\right|\left|\int_{-\infty}^{\infty}(-i y)^{m-4} K(y)^{-1} \exp \left(-t y^{2}-i y(s-u)\right) d y\right|
$$

converges and is a continuous function of $x$. By Fubini's theorem the order of the outer two integrations in (8) may be interchanged so that

$$
\begin{aligned}
\beta_{t}^{m}(s)= & (2 \pi)^{-3 / 2} \lim _{A \rightarrow \infty} \int_{-A}^{A} d \alpha(x) \int_{-\infty}^{\infty} k^{(4)}(u-x) d u \\
& \cdot \int_{-\infty}^{\infty}(-i y)^{m-4} K(y)^{-1} \exp \left(-t y^{2}-i y(s-u)\right) d y .
\end{aligned}
$$

A trivial estimate now shows that the inner two integrals may be interchanged yielding

$$
\begin{aligned}
& \beta_{t}^{m}(s)=(2 \pi)^{-3 / 2} \lim _{A \rightarrow \infty} \int_{-A}^{A} d \alpha(x) \\
& \cdot \int_{-\infty}^{\infty}(-i y)^{m-4} K(y)^{-1} \exp \left(-t y^{2}-i y s\right) d y \int_{-\infty}^{\infty} k^{(4)}(u-x) e^{i y u} d u .
\end{aligned}
$$


Since

$$
\begin{aligned}
(2 \pi)^{-1 / 2} \int_{-\infty}^{\infty} k^{(4)}(u-x) e^{i y u} d u & \\
& =e^{i y x}(2 \pi)^{-1 / 2} \int_{-\infty}^{\infty} k^{(4)}(u) e^{i y u} d u=e^{i y x}(-i y)^{4} K(y),
\end{aligned}
$$

we conclude that

$$
\begin{aligned}
\beta_{t}^{m}(s) & =(2 \pi)^{-1} \int_{-\infty}^{\infty} d \alpha(x) \int_{-\infty}^{\infty}(-i y)^{m} \exp \left(-t y^{2}+i y(x-s)\right) d y \\
& =(4 \pi t)^{-1 / 2} \int_{-\infty}^{\infty} \frac{d^{m}}{d s^{m}} \exp \left[-\frac{(s-x)^{2}}{4 t}\right] d \alpha(x)=\tilde{\alpha}_{t}^{(m)}(s),
\end{aligned}
$$

which is the statement of Theorem 1.

We now turn to Theorem 2. If we choose $\beta_{t}(s)$, a particular $m$ th integral of $\beta_{t}^{m}(s)$ for each $t$, then we know that this $\beta_{t}(s)$ must differ from $\alpha_{t}(s)$ by at most a polynomial of degree $m-1$. Call this polynomial $p_{t}^{m-1}(s)$ and consider the equation

$$
\tilde{\alpha}_{t}(s)=\beta_{t}(s)-p_{t}^{m-1}(s) .
$$

Our problem is to find the function $p_{t}^{m-1}(s)$ for all $t$ (or at least for a sequence $t_{n} \rightarrow 0+$ ). Let

$$
H_{t}(y)=(4 \pi t)^{-1 / 2} \int_{-\infty}^{\infty} \exp \left(-(y-u)^{2} / 4 t\right) f^{\prime}(u) d u .
$$

Then from (4)

$$
\begin{gathered}
H_{t}(y)=(4 \pi t)^{-1 / 2} \int_{-\infty}^{\infty} \exp \left(-(y-u)^{2} / 4 t\right) d u \\
\cdot \lim _{A, B \rightarrow \infty} \int_{-A}^{B} k^{\prime}(s) d_{s} \alpha(u-s) .
\end{gathered}
$$

As in the proof of (6) the convergence of the outer integral is uniform in $A$ and $B$ so that

$$
\begin{gathered}
H_{t}(y)=\lim _{A, B \rightarrow \infty} \int_{-A}^{B} k^{\prime}(s) d_{s}(4 \pi t)^{-1 / 2} \int_{-\infty}^{\infty} \exp \left(-(y-u)^{2} / 4 t\right) \alpha(u-s) d u \\
=\lim _{A, B \rightarrow \infty} \int_{-A}^{B} k^{\prime}(s) d s(4 \pi t)^{-1 / 2} \\
\cdot \int_{-\infty}^{\infty} \frac{d}{d s} \exp \left(-(y-s-v u)^{2} / 4 t\right) \alpha(v) d v
\end{gathered}
$$


If we first integrate the inner integral by parts and then the outer one we obtain

$$
\begin{aligned}
H_{t}(y) & =\lim _{\boldsymbol{A}, B \rightarrow \infty} \int_{-\boldsymbol{A}}^{B} k(s) d_{s} \tilde{\alpha}_{t}(y-s) \\
& =\lim _{\boldsymbol{A}, B \rightarrow \infty} \int_{-A}^{B} k(y-s) d \tilde{\alpha}_{t}(s) .
\end{aligned}
$$

It follows that the limits in (11) exist and, in fact, that all the conditions necessary for the proof of Lemma 1 now also hold for $\tilde{\alpha}_{t}(s)$ so that $\tilde{\alpha}_{t}(s)=o\left(s^{n}\right)$ for each $t>0$. If this result is combined with (9) it is clear that at least the higher powers in $p_{t}^{m-1}(s)$ are uniquely determined. If we write

$$
p_{t}^{m-1}(s)=p_{t}^{n-1}(s)+\sum_{j=n}^{m} c_{t}^{j} s^{j},
$$

then in order for (9) and $\tilde{\alpha}_{t}(s)=o\left(s^{n}\right)$ to be satisfied the $c_{t}^{J}$ must be determined by

$$
\begin{aligned}
\lim _{s \rightarrow \infty} s^{-m} \beta_{t}(s) & =c_{t}^{m}, \\
\lim _{\delta \rightarrow \infty} s^{-m+1}\left[\beta_{t}(s)-c_{t}^{m} s^{m}\right] & =c_{t}^{m-1}, \text { etc. }
\end{aligned}
$$

Now let

$$
\bar{\beta}_{t}(s)=\beta_{t}(s)-\sum_{j=n}^{m} c_{t}^{j j}
$$

so that

$$
\tilde{\alpha}_{t}(s)=\bar{\beta}_{t}(s)-p_{t}^{n-1}(s) .
$$

Substitute this in (11) to obtain

$$
H_{t}(y)=\int_{-\infty}^{\infty} k(y-s) d \beta_{t}(s)-\int_{-\infty}^{\infty} k(y-s) d p_{t}^{n-1}(s) .
$$

Since $k(y)=O\left(|y|^{-n}\right)$ the second of these integrals exists so that the first one does also. Moreover since $H_{t}(y)$ is determined by the definition (10) and $\bar{\beta}_{t}(s)$ is determined from (13), equation (14) is in effect an equation for $\int k(y-s) d p_{t}^{n-1}(s)$ which is a polynomial of degree not greater than $n-2$. This means that

$$
H_{t}(y)-\int_{-\infty}^{\infty} k(y-l s) d \bar{\beta}_{t}(s)
$$


is a known polynomial of degree not greater than $n-2$. Therefore the coefficients of the polynomial

$$
\int_{-\infty}^{\infty} k(y-s) d p_{t}^{n-1}(s)=\sum_{j=1}^{n-1} j c_{t}^{j} \int_{-\infty}^{\infty} k(u)(y-u)^{j-1} d u
$$

are uniquely determined. It is clear from the right side of (15) that this determines the $c_{t}^{j}$ uniquely. Theorem 2 is therefore complete.

The last theorem follows almost immediately. Once

$$
\tilde{\alpha}_{t}(s)=\beta_{t}(s)-p_{t}^{m-1}(s)=\left(4 \pi_{t}\right)^{-1 / 2} \int_{-\infty}^{\infty} \exp \left(-(u-s)^{2} / 4 t\right) d \alpha(u)
$$

is known $\alpha(u)$ can easily be found. Integrating by parts and then integrating from 0 to $s$ we find

$$
\begin{aligned}
\int_{0}^{s} \tilde{\alpha}_{t}(s) d s= & (4 \pi t)^{-1 / 2}\left\{\int_{-\infty}^{\infty} \exp \left(-(u-s)^{2} / 4 t\right) \alpha(u) d u\right. \\
& \left.-\int_{-\infty}^{\infty} \exp \left(-u^{2} / 4 t\right) \alpha(u) d u\right\} .
\end{aligned}
$$

From this one concludes in the usual way that

$$
\lim _{t \rightarrow 0+} \int_{0}^{s} \tilde{\alpha}_{t}(s) d s=2^{-1}(\alpha(s+)+\alpha(s-))-\alpha(0)=2^{-1}(\alpha(s+)+\alpha(s-)) .
$$

\section{REFERENCES}

1. I. I. Hirschman and D. V. Widder, The convolution transform, Princeton University Press, 1955.

2. H. Pollard, The Poisson transform, Trans. Amer. Math. Soc. vol. 78 (1955) pp. 541-550.

Syracuse University 